not yet available for HMTSF-TCNQ (or HMTTF-TCNQ).

\section{Conclusion}

We have shown how the properties of quasi-one-dimensional metals are dominated by the values of the collision time along the chain, $\tau_{\|}$and the interchain tunnelling matrix element, $t_{\perp}$. A true one-dimensional metal is defined by the relations

$t_{\perp}<\hbar / \tau_{\|}<E_{F}$
As $\hbar / \tau_{\|} t_{\perp}$ diminishes, a transition to an anisotropic, three-dimensional, high mobility semi-metal takes place. This Diffusive to Coherent Transition (alias, the opening of a covalency gap) does not have a significant effect on the longitudinal and transverse conductivities, but greatly affects the Hall constant, magnetoresistance, diamagnetic susceptibility, EPR line width, NMR inter-chain tunnelling time, the Peierls transition temperature $T_{p}$ and its pressure dependence, and the sensitivity of the resistivity to radiation damage.

Thus, we have a unified phenomenological description of a very wide variety of phenomena, which does not depend upon the microscopic origin of the collision time $\tau_{\|}$.

\section{References}

1. "Low Dimensional Co-operative Phenomena", Editor H.J. Keller, NATO AS1 series B7, (Plenum Press, New York) 1975 2. "Chemistry and Physics of One-Dimensional Metals", Editor H.J. Keller, NATO AS1 series B25, (Plenum Press, New York) 1977

3. "Organic Conductors and Semiconductors", Conference Proceedings, Siofok, Hungary, 1976, Lecture Notes in Physics, 65. (Springer Verlag) 1977

4. SODA, G., JEROME, D., WEGER, M., ALIZON, J., GALLICE, J., ROBERT, H., FABRE, J.M. and GIRAL, L. J. Physique 38 (1977) 931

5. PIPPARD, A.B. Reports on the Progress of Physics 23 (1960) 176

\title{
Upsilon Particle Produced at Desy
}

The upsilon-particle, the first signs of which were seen in proton-nucleus collisions, has now been produced for the first time in electron-positron collisions, where it can be studied with more precision. The rate at which the new particle is produced and its long life-time indicate that it is very probably a compound, made up of two quarks of a new type. Although the quarks are new, the experiments support the general quark picture surprisingly well.

The experiments were performed at the $\mathrm{e}^{+} \mathrm{e}^{-}$storage-ring DORIS, modified substantially to reach the energy of the upsilon particle. Orinally, DORIS had been designed for a total centre of mass energy of 7 $\mathrm{GeV}$, obtained in the collision of two beams of $3.5 \mathrm{GeV}$ each, but the 48 bending magnets had fortunately been built powerful enough to deal with beams of up to about $4.5 \mathrm{GeV}$ and to obtain the necessary RF power, cavities from the storage ring PETRA (now under construction) were substituted for the normal cavities. For the upsilon search, the total energy of DORIS was upgraded to $9.5 \mathrm{GeV}$.

The measurements of the upsilon were carried out with the doublearm-spectrometer DASP and the detector PLUTO incorporating a superconducting magnet. This has been improved substantially over the past year to cover more than $96 \%$ of the solid angle by a system of shower counters, which now permit the detection of photons in addition to charged particles.

The first result from the DESY teams * was the exact determination of the mass of the upsilon which came out to be $(9.46 \pm .01) \mathrm{GeV} / \mathrm{c}^{2}$; the width of the mass-distribution was too small to be measured, indicating therefore a long life-time. This fact agrees with the hypothesis that the upsilon is a relatively stable compound state of two new quarks, each assumed to have a mass around $5 \mathrm{GeV} / \mathrm{c}^{2}$. According to current theories, the DESY data show that the new quarks have an electric charge of $1 / 3 \mathrm{e}$. The characteristic property of these quarks is a new quantum-number called (arbitrarily) "beauty" (to add to the existing quantum numbers: "up", "down", "strange", and "charm ").

During the scan for the upsilon another very important discovery was made: particle production at these energies proceeds mainly via an intermediate state which is believed to be a high energy quark-antiquark pair. The two quarks immediately transform into stable particles which are then detected as two characteristic "jets". This fact is another important piece of evidence in favour of the hypotheses of the quark structure of matter. In addition, these jet events will provide important information to check the quark theory in detail and to learn about the forces acting between quarks.

\footnotetext{
* The two measuring teams included physicists from DESY, the Universities of Dortmund, Hamburg, Heidelberg and Lund, the Physics Institute of the RWTH at Aachen, the Gesamthochschules of Siegen and of Wüppertal, and included also physicists on detachment from the Universities of Tel Aviv, Humboldt and Purdue.
}

\section{Albert Einstein Centenary}

\section{IN HONOUR OF THE ONE HUNDRETH ANNIVERSARY OF THE BIRTH OF ALBERT EINSTEIN}

SPONSORED BY: UNESCO, IUPAP, INT. COM. ON GRG, CERN, SWISS ACAD. OF SCIENCES, UNIVERSITY OF BERN, THE SWISS AUTHORITIES, THE ALBERT EINSTEIN FOUNDATION

\section{A PUBLIC CONFERENCE 13-17 MARCH, 1979 \\ in the Alfa Conference Centre BERN}

with the participation of the world's leading theoretical physicists.

No registration fee will be charged.

BUT prior registration is necessary. Please use form opposite.
Complete and return the form below by 30 Sept., to: Dr. Alan Held, Dept. of Theor. Phys., Sidlerstrasse 5, CH-3012 BERN.

Name :

First Name :

Address :

\begin{tabular}{|c|c|}
\hline Tel. : & Telex: \\
\hline Accom & \\
\hline Date : & Sig. : \\
\hline
\end{tabular}

